\title{
Tasquinimod: a novel drug in advanced prostate cancer
}

Susanne Osanto*1, Hendrik van Poppel ${ }^{2}$ \& Jacobus Burggraaf ${ }^{3}$

Department of Clinical Oncology, Leiden University Medical Center, Leiden, 2333ZA, The Netherlands 2Department of Urology, KU Leuven, Leuven, B-3000, Belgium

${ }^{3}$ Centre for Human Drug Research, Zernikedreef 8, Leiden, 2333CL, The Netherlands

*Author for correspondence: s.osanto@lumc.nl

Tasquinimod, an oral quinolone-3-carboxamide with anti-tumor activity in preclinical models of prostate cancer, has been tested in patients with minimally symptomatic castration-resistant prostate cancer (CRPC), showing promising inhibitory effects on the occurrence of metastasis and delayed disease progression. Although its mode of action is not fully understood, tasquinimod presumably exerts its unique anti-tumor action through inhibition of angiogenesis and immunomodulation. In clinical studies, tasquinimod demonstrated anti-tumor activity in prostate cancer in combination with a mild-to-moderate side effect profile. With single-agent tasquinimod, dose-limiting toxicity was amylase elevation without signs of pancreatitis and sinus tachycardia. The maximum tolerated dose in Phase I studies in patients with CRPC was once daily administration of 0.5-1-mg tasquinimod orally. In a Phase II trial, significant clinical activity has been demonstrated in asymptomatic or minimally symptomatic, chemotherapy-naive, metastatic CRPC (mCRPC) patients. Men were randomized to tasquinimod or placebo in a 2:1 fashion; treatment with tasquinimod resulted in significant improvement of median progression-free survival (7.6 vs 3.3 months with placebo; $p=0.0042$ ). Based on these encouraging effects, a randomized, double-blind, placebo-controlled trial in men with minimally symptomatic mCRPC has been designed. This large Phase III trial, powered for a primary end point of progression-free survival, has now enrolled the target number of 1200 men. If the Phase II data are validated in the Phase III trial a new compound with a unique mode of action might become approved as a future therapy for minimally symptomatic mCRPC patients.

Prostate cancer is the most common nonskin cancer in men in northern European countries and the USA and the second leading cause of cancer-related death in men in most western countries [1-4]. The 2008 projected number of new cases worldwide was 914,000 , with an estimated 258,000 deaths [101]. Most men treated for localized prostate cancer are cured, but a third of patients will experience recurrence of the disease.

Prostate cancer recurrence is usually detected by a rise in prostate-specific antigen (PSA). A large number of these patients may remain asymptomatic or mildly symptomatic for years.

After a rising PSA has been detected, initiation of androgen deprivation therapy has been the standard of care in advanced prostate cancer for decades. Androgen deprivation is effective in the vast majority of patients for several years, but almost all will eventually develop progressive disease (castration-resistant prostate cancer; CRPC) despite low levels of testosterone.

Until recently therapeutic options for patients with metastatic CRPC (mCRPC) were limited with only docetaxel as the standard first-line treatment. Patients with recurrence but minimal tumor burden were usually observed in order to postpone the start of chemotherapy until the patient developed symptoms or symptomatic disease was impending.

The landscape of metastatic prostate cancer has rapidly changed in the last 2-3 years and novel agents have recently been added to the current armamentarium based on Phase III trials. Since 2010, a number of agents have been approved for the treatment of CRPC patients based on overall survival (OS) benefit [5]. Currently, Phase III studies have shown a survival advantage for cabazitaxel, abiraterone, enzalutamide, radium-223 and sipuleucel- $T$ in the post- and pre-docetaxel space [6-12].

Angiogenesis is a hallmark of cancer, and the development of angiogenesis inhibitors seemed logical and relevant. Until now antiangiogenesis agents tested in Phase II and subsequently in Phase III trials in combination with docetaxel have not been able to prolong OS compared with docetaxel plus prednisone alone. Perhaps those patients have more advanced disease

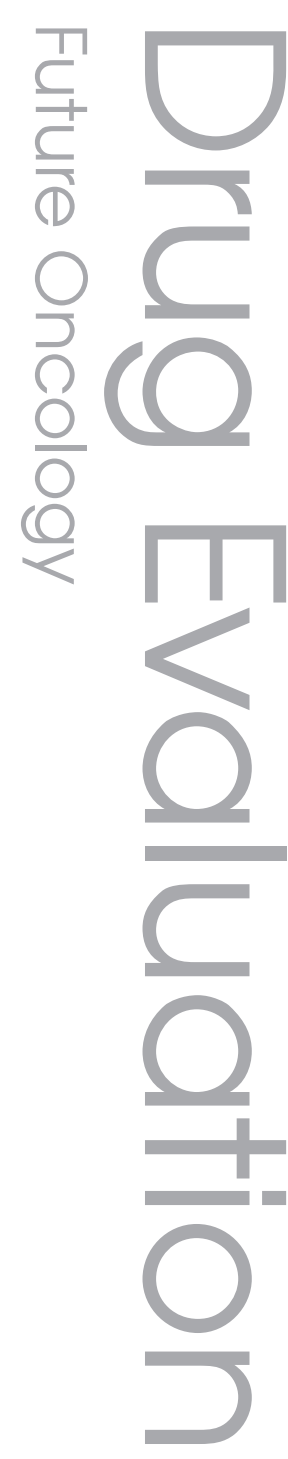

\section{Keywords}

- antiangiogenic agent - castration-resistant prostate

cancer $=$ prostate cancer

- quinoline-3-carboxamide - tasquinimod

\section{Future : Medicine $\stackrel{\text { part of }}{ }$}


with bone metastases, which are less suitable for antiangiogenic agents.

Currently, novel compounds with different modes of action are under investigation in various stage settings of CRPC. There is in particular an unmet clinical need to develop active, nontoxic therapies for minimally symptomatic CRPC patients. It appears that targeting angiogenesis could be particularly attractive owing to the relatively low tumor burden. If successful, it would provide a window of opportunity that offers at least the possibility to postpone the generally more toxic chemotherapy.

\section{Tumor angiogenesis}

Angiogenesis occurring around tumors was described more than 100 years ago. Subsequently in the late 1960s, tumors were suggested to produce a diffusible angiogenic substance $[13,14]$. Folkman reported a factor called tumorangiogenesis factor and suggested that blockade of this factor might arrest solid tumors at a tiny diameter of a few millimeters. His observation that solid tumors are angiogenesis dependent for their tumor growth [15] was received with skepticism and it took years before the concept of angiogenesis became widely accepted.

Gullino demonstrated that premalignant cells acquire angiogenic capacity before turning into malignant cells and proposed that this concept be used to design strategies to prevent cancer [16].

Various efforts were critically important to the further development of this field. Assays for angiogenesis were developed and long-term culture of capillary endothelial cells was established. Pro- and antiangiogenic factors were purified from malignant and non-malignant endothelial and stromal cells, and were shown to regulate angiogenesis. Nonendothelial cells, including mast cells, were shown to modulate angiogenesis. Animal studies demonstrated that tumors may regress and completely disappear upon treatment with angiogenesis inhibitors.

Tumor angiogenesis with its disorganized and irregular structured angiogenic vessels with abnormal blood flow, is now being recognized as a hallmark of advanced cancers and an attractive treatment target in multiple solid tumors $[17,18]$.

Based on preclinical data, several antiangiogenic agents alone or in combination with conventional therapies are in clinical trials or are already registered for the treatment of a particular type of cancer, for example, angiogenesis inhibitors targeting the VEGF signaling pathways in renal cell cancer.

\section{Tasquinimod: a novel quinoline}

Tasquinimod (Figure 1) is a novel small-molecule inhibitor and second-generation oral quinoline3-carboxamide analog with antiangiogenic properties and tumor growth-inhibiting activity against human prostate cancer models. Its mechanism of angiogenesis inhibition is not fully understood. Quinolines are classified based on the number of substituants present on the quinoline ring or compounds containing a quinoline ring fused to other heterocycles.

\section{Mechanism of action in preclinical studies}

The quinoline-3-carboxamide, linomide, has anti-tumor effects against a wide variety of experimental tumors in animal studies, including prostate cancer, and the anticancer effects of linomide have been shown to be mediated via an antiangiogenic response [19,20]. Linomide was tested in a series of Phase II and III trials in patients with advanced renal cell carcinoma and with multiple sclerosis. The oral quinoline compound tasquinimod is structurally related to linomide, but has 30- to 60-times more antitumor potency [21]. Its potent activity against human prostate cancer xenografts [21-24] and interference with the metastatic process [25] is presumably based on inhibition of tumor establishment (Table 1).

Inhibition of angiogenesis by tasquinimod has been demonstrated in several in vitro assays of endothelial capillary tube formation, aortic ring and chorioallantoic membrane assay [21]. The antiangiogenic effects of tasquinimod are not mediated via the blockade of VEGF or inhibition of VEGF receptor tyrosine kinases [21]. Tasquinimod is a pleiotropic compound with immunomodulatory, antiangiogenic and antimetastatic activity in human prostate cancer animal models. Tasquinimod induces upregulation of the endogenous angiogenesis

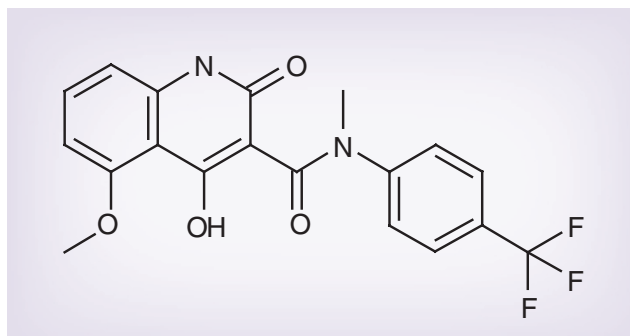

Figure 1. Tasquinimod. Structure: 4-hydroxy5-methoxy-N,1-dimethyl-2-oxo- $N$-(4[trifluoromethyl] phenyl)-1,2-dihydroquinoline3-carboxamide; formula: $\mathrm{C}_{2} \mathrm{OH}_{17} \mathrm{~F}_{3} \mathrm{~N}_{2} \mathrm{O}_{4}$; molecular mass: $406.355 \mathrm{~g} / \mathrm{mol}$. 


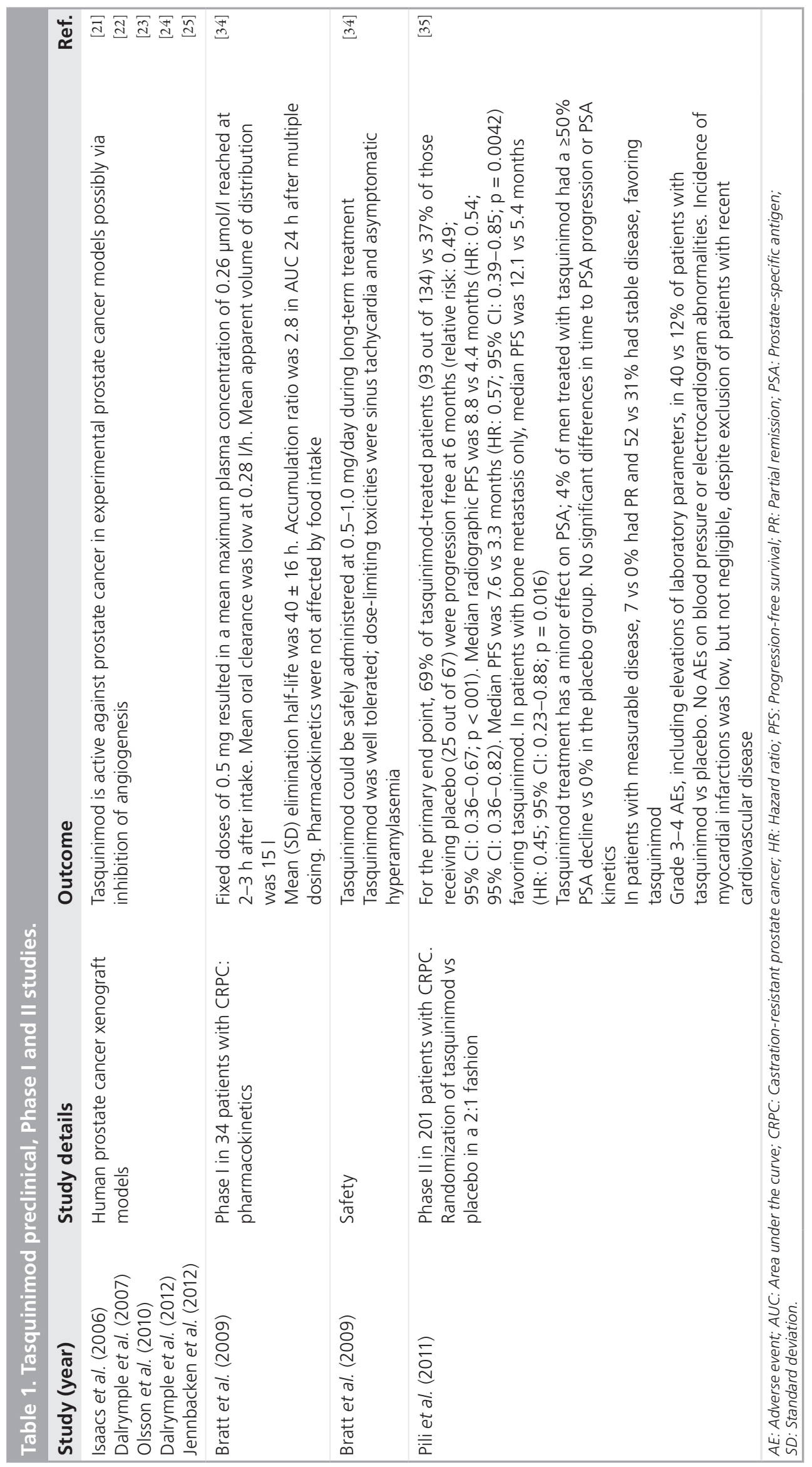


inhibitor TSP-1 [23,26] and downregulation of HIF-1, and $\alpha$-subunit-regulated proteins, which in turn leads to reduced angiogenesis via inhibition of the 'angiogenic switch'. Tasquinimod targets and strongly binds to S100A9 (MRP-14, a protein in the family of calcium-binding S100 proteins), an immunomodulatory protein expressed on myeloid-derived suppressor cells present in the tumor microenvironment that promotes tumor development via VEGF-dependent and -independent pathways [27-32].

Tumor growth is impaired in S100A9 knockout mice [33], suggesting that S100A9 is indeed a relevant therapeutic target. Available preclinical data do not support the use of serum PSA as an indicator of therapeutic response.

Various human prostate cancer xenograft models, which were either prostate cancers that expressed the androgen receptor (AR; AR positive) or did not (AR negative), or expressed the wild-type androgen receptor (AR wild-type) or a mutant androgen receptor (AR mutant). In addition, tumors varied with regard to expression of PSA, (i.e., PSA-positive or PSA-negative tumors). These aforementioned human prostate cancer xenografts were shown to be responsive to treatment with tasquinimod, resulting in a significantly decreased tumor growth rate and volume. However, tasquinimod does not directly affect PSA levels in either of the xenograft models tested. Based on its efficacy seen in prostate-cancer bearing animal studies, tasquinimod has been developed for clinical application as a treatment for prostate cancer.

\section{Pharmacokinetics}

The pharmacokinetics of tasquinimod were evaluated in patients with histologically or cytologically proven CRPC with PSA progression and a castrate level of testosterone in two open-label dose-escalation studies (Table 1) [34]. Patients had not received prior chemotherapy and, when applicable, treatment with a luteinizing hormone-releasing hormone agonist for androgen suppression was continued.

\section{Phase I (study 1)}

In the first study a cohort of six eligible patients were initially given a dose of $0.5 \mathrm{mg}$ once daily. If the patients tolerated this dose, the next cohorts, each comprising six patients, were to receive escalating doses (1.0, 2.0 and $3.0 \mathrm{mg} / \mathrm{day})$ of tasquinimod until the maximum tolerated dose (MTD). The patients were treated for 28 days, followed by a 1-week off-period, and during an extension study thereafter for up to 12 months.
The MTD was defined as the highest dose level at which no more than one out of six patients experienced a dose-limiting toxicity (DLT). Any drug-related grade 3 or 4 toxicity was considered a DLT [34].

\section{Phase I (study 2)}

The second study was an intrapatient stepwise dose-escalation study in eight patients. During Phase I evaluation, men with CRPC were initially treated with a once-daily oral dose of $0.25 \mathrm{mg}$ for 3 weeks, with gradual dose escalation to a MTD of $1.0 \mathrm{mg} /$ day over an additional 4 weeks. In the absence of treatment-related toxicity, patients were offered to continue their treatment at $1.0 \mathrm{mg} /$ day during an extension study for another 11 months [34].

\section{Plasma pharmacokinetics}

In study 1, extensive pharmacokinetic sampling was performed on days 1 and 28. A blood sample (predose) was collected at all visits. Tasquinimod concentrations in plasma samples were determined using a validated liquid chromatography with tandem mass spectrometry detection.

It was shown that fixed doses of $0.5 \mathrm{mg}$ resulted in mean maximum plasma concentrations of $0.26 \mu \mathrm{mol} / \mathrm{l}$ that were reached at $2-3 \mathrm{~h}$ after intake. Mean oral clearance was low at $0.28 \mathrm{l} / \mathrm{h}$ and the mean apparent volume of distribution was $15 \mathrm{l}$. The mean and standard deviation elimination half-life was $40 \pm 16 \mathrm{~h}$ and this translated into an accumulation ratio of 2.8 in area under the curve $24 \mathrm{~h}$ after multiple dosing. The pharmacokinetics seem linear over the $0.25-1.0-\mathrm{mg}$ dose range and apparently are not affected by food intake. There are no indications for time dependency in exposure after long-term oral dosing as steady state concentrations at 12 months were the same as after 14 days of administration. Preclinical data obtained after treatment periods of at least 10 days in different tumorbearing mice strains showed that the wide range (between 1-30 mg/kg daily) of doses employed, resulted in highly comparable maximal plasma levels in the order of $2-4 \mu \mathrm{M}$ [22]. The maximal plasma concentrations are an order of magnitude higher compared with the maximal concentration observed of $0.3 \mu \mathrm{M}$ in humans [34]. Thus, substantial differences exist in the pharmacokinetics of the compound between rodents and humans, as can also be concluded from the differences in the elimination half-life that was also an order of magnitude shorter in rodents $(3.4 \mathrm{~h})$ compared with humans $(40 \mathrm{~h})$. It also appears that in patients, substantially lower 
doses are needed compared with animals. This only reflects pharmacokinetic differences; differences in the blood:tumor ratio or sensitivity are presently unknown.

Unfortunately, there are no data available regarding potential drug-drug interactions as patients using CYP3A4 inhibitors or inducers were excluded from the early studies and no reports are available on this topic in the public domain. In addition, information on possible interactions with the efflux transporter p-glycoprotein are currently lacking.

\section{Safety results}

Inflammation $(41 \%)$ was the most common adverse event (AE) associated with tasquinimod in the fixed 0.5-mg dose group during the first 4 weeks of treatment. In this study, inflammation is defined as increased levels of laboratory markers, constituting laboratory changes above the normal upper limit not associated with clinical symptoms requiring any clinical intervention. In the fixed 1.0-mg dose group, nausea (57\%) and fatigue (43\%) were the most common AEs. The majority of these events were grade 1 in severity, transient in accordance with the common toxicity criteria (CTC) and occurred early after the start of treatment (TABLE 1) [34].

\section{Adverse effects}

In these Phase I studies, tasquinimod could be safely administered at $0.5-1.0 \mathrm{mg} /$ day during long-term treatment. Most AEs occurred within the first 4 weeks of treatment. Of a total of 71 related AEs in the fixed $0.5-\mathrm{mg}$ dose group, $76 \%$ were recorded until day 35 . Raised inflammatory markers not associated with clinical symptoms are the most frequent AEs, whereas fatigue, myalgia and nausea are the most common symptomatic AEs. Several patients developed asymptomatic elevations in amylase approximately 1 week after the start of treatment. Amylase levels normalized without dose interruption within a couple of weeks. The reasons for this transient elevation in amylase are unknown, as hyperamylasemia was not associated with clinical symptoms and no patient developed acute pancreatitis [34].

\section{Maximum tolerated dose}

In study one, 24 patients received tasquinimod at fixed doses of 0.5 (17 patients) or $1.0 \mathrm{mg}$ (seven patients) orally once daily on a continuous dosing schedule for 28 days. Patients treated at the $0.5-\mathrm{mg}$ dose level tolerated the therapy without DLT. In the oral $1 \mathrm{mg}$ daily cohort, two patients experienced DLT. One patient had an asymptomatic CTC grade 3 elevation in amylase on day 23. He recovered without any clinical intervention within 2 weeks. The second DLT patient had chest and back pain and was hospitalized on day 10 owing to a grade 3 cardiac arrhythmia corresponding to supraventricular tachycardia. There were no signs consistent with myocardial infarction. He received treatment with $\beta$-blockers and recovered 2 weeks later. Owing to these observations, the remaining patients in the 1.0-mg dose group were discontinued prematurely, and the MTD of tasquinimod was determined to be $0.5 \mathrm{mg}$ once daily [34].

In study two (evaluating safety of $1-\mathrm{mg} /$ day tasquinimod after dose escalation) three serious AEs possibly related to tasquinimod were observed: musculoskeletal pain grade 2; chest pain grade 3; and cerebral infarction grade 3 [34].

\section{Efficacy}

Efficacy was assessed by measuring PSA levels in all patients and by bone scans in patients treated at $0.5 \mathrm{mg}$ per day. A serum PSA decline of $\geq 50 \%$ was noted in two patients. The median time to PSA progression ( $>25 \%)$ was 19 weeks. PSA progression-free status was evaluated at 18 weeks and not at 12 weeks, which would be according to Prostate Cancer Working Group (PCWG2) criteria. Of the 32 patients, 56\% were progression free by PSA criteria ( $<25 \%$ increase) at 18 weeks. Only two patients experienced a serum PSA decline of $\geq 50 \%$. The median time to PSA progression ( $>25 \%)$ was 19 weeks. The median PSA-doubling time at 18 weeks was 65 weeks compared with the median pretreatment PSA-doubling time of 25 weeks [34].

Repeated radiological imaging with bone scans were performed in patients who received the fixed dose of $0.5 \mathrm{mg}$ per day. Seven of these patients had a positive bone scan at baseline, whereas eight patients had negative scans. Only three (20\%) out of 15 patients (median time on study was 34 weeks) had radiographic progression on bone scans with the development of new bone lesions. Two of these patients had negative bone scans at baseline [34].

\section{Tasquinimod in a Phase II trial in prostate cancer patients}

The effect of tasquinimod on delaying disease progression in CRPC was explored in a Phase II trial.

A randomized, double-blind, placebo-controlled multicenter Phase II study comparing tasquinimod 
with placebo in 206 men with $\mathrm{mCRPC}$ and minimal symptoms was completed in 2009 (TABLE 1) [35].

Patients were randomized in a doubleblind 2:1 fashion between tasquinimod (oral $0.25 \mathrm{mg} /$ day once daily, escalating to $1.0 \mathrm{mg} /$ day once daily for 4 weeks) or placebo. Patients received a once-daily oral dose of $1.0-\mathrm{mg}$ tasquinimod or a placebo after a titration phase $(0.25 \mathrm{mg} /$ day for 2 weeks followed by $0.5 \mathrm{mg} /$ day for 2 weeks), leading to a maximum of 6 months of double-blind treatment. Asymptomatic patients in the placebo group with disease progression during the first 6 months or without progression at 6 months were offered open-label tasquinimod. Patients on tasquinimod with no disease progression at 6 months were offered open-label treatment until progression; patients with disease progression were withdrawn.

The primary end point was the proportion of patients without disease progression at 6 months, defined by response evaluation criteria in solid tumors and PCWG2 criteria [36], or pain criteria, excluding PSA. Progression-free survival (PFS) was defined as time from first dose to first progression or death.

Eligibility criteria included: histologically confirmed prostate adenocarcinoma, Karnofsky performance score (KPS) of 70-100, castrate levels of testosterone $(\leq 50 \mathrm{ng} / \mathrm{dl})$, visual analog scale pain score of 3 or greater (scale: $0-10$ ), and radiologically confirmed metastatic disease with progression defined by rising serum PSA levels (confirmed by three consecutive measurements within 1 year at least 14 days apart), progression of bidimensionally measurable soft-tissue metastasis or new bone lesions detected by bone scan within 12 weeks before screening.

Men with a history of pancreatitis or cardiovascular disease including recent ( $<12$ months) myocardial infarction, congestive heart failure, ventricular arrhythmias or unstable angina were excluded. Concomitant use of a stable dose of an antiandrogen was permitted, provided that criteria for progression were met. Patients were stratified based on KPS into KPS: 90-100 vs 70-80).

Between December 2007 and June 2009, 206 men were enrolled at 45 centers in the USA, Canada and Sweden. Groups were imbalanced for some baseline characteristics, such as the presence of tumor pain (28 vs $11 \%$ ), and visceral metastases (24 vs 15\%), which were more common in the tasquinimod group, which also had higher median baseline PSA levels, shorter median PSA doubling time and a higher proportion of African-American patients (15 vs 3\%).
Two hundred and one men (134 tasquinimod and 67 placebo) with a median age of 72.5 years were evaluable for efficacy and safety.

\section{Pharmacokinetics}

Pharmacokinetic analyses were performed in all patients at 2, 4, 8, 12, 24, 36 and 48 weeks after treatment initiation as described by Bratt et al. [34]. These investigations confirmed the previous findings on clearance and apparent volume of distribution reported previously [37]. However, there appears to be a slight increase in clearance with increasing dose, and the data also suggested that tasquinimod clearance decreased by $1.4 \%$ per year of age. The latter finding might be important as it correlated with a higher discontinuation rate with increasing age. There are no indications that hepatic function and race/ethnicity have a substantial influence on the pharmacokinetics of tasquinimod.

\section{Efficacy}

Median PFS was 7.6 months for patients treated with tasquinimod and 3.3 months for patients treated with placebo $(\mathrm{p}=0.0042)$. After 6 months of treatment, only $31 \%$ of the 134 patients on tasquinimod had progressed versus $63 \%$ of the 67 patients in the placebo group (hazard ratio [HR]: 0.49; 95\% CI: 0.36-0.67; $\mathrm{p}<0.001)$. Bone alkaline phosphatase levels were stabilized in the tasquinimod group, but no significant differences in time to PSA progression or in PSA kinetics were observed between the two treatment groups. PSA response to tasquinimod was minor with only $4 \%$ of men treated with tasquinimod showing a $50 \%$ or greater PSA decline versus $0 \%$ in the placebo group.

In the double-blind phase, $84 \%$ of patients with progressive disease had radiographic progression, whereas symptomatic or clinical progression was documented in 22\% of patients. Progression on bone scan was more common in the placebo group (26 vs $15 \%$ of progression events). Median radiographic PFS was 8.8 versus 4.4 months (HR: 0.54 ; 95\% CI: 0.36-0.82) favoring tasquinimod.

In addition, subset analysis of patients was performed according to PCWG2 CRPC subtypes defined by the localization of metastatic lesions. For men with visceral metastases, bone metastases with or without nodal metastases, and lymph node-only metastases, median PFS in tasquinimod-treated men versus those receiving placebo was 6.0 versus 3.0 months (HR: 0.41 ; $95 \%$ CI: $0.16-1.02 ; \mathrm{p}=0.045)$, 8.8 versus 3.4 months (HR: 0.56; 95\% CI: 0.34-0.92; 
$\mathrm{p}=0.019)$ and 6.1 versus 3.1 months (HR: 0.73; 95\% CI: 0.27-2.00; $\mathrm{p}=0.54$ ), respectively. Median PFS in men with bone metastases only at baseline was 12.1 versus 5.4 months (HR: 0.45; 95\% CI: 0.23-0.88; $\mathrm{p}=0.016$ ).

Independently scored radiologic responses were 3\%: partial response; 58\%: stable disease; and 39\%: progressive disease in patients assigned to tasquinimod arm versus $0 \%$ : partial response; 35\%: stable disease; and 65\%: progressive disease in patients assigned to the placebo arm.

\section{Safety \& AEs}

In general, tasquinimod treatment was safe. The most frequently reported AEs were fatigue, gastrointestinal disorders (nausea, constipation and flatulence), musculoskeletal pain and transient increases in certain laboratory parameters. AEs occurring more frequently in the tasquinimod arm included GI tract disorders, fatigue, musculoskeletal pain, and elevations of pancreatic and inflammatory biomarkers. Grade 3-4 AEs, including asymptomatic elevations of laboratory parameters, were reported in $40 \%$ of patients receiving tasquinimod versus $12 \%$ receiving placebo; deep vein thrombosis (4 vs $0 \%$ ) was more common in the tasquinimod arm. In most cases the AEs were mild or moderate, transient and reversible, and more severe cases could be managed by dose reductions or supportive measures, with continuation of drugs at individual MTDs. The number of AEs was higher during the first weeks of treatment. The Phase II trial had more men older than 80 years of age (22 vs $6 \%$ in Phase I). Tolerability decreased with age which may be explained by a decreased hepatic clearance of tasquinimod in elderly patients. Importantly, cardiovascular event rates were higher in tasquinimod-treated patients, although men with prior recent cardiovascular disease were excluded. The number of myocardial infarctions was low, no AEs on blood pressure or electrocardiogram abnormalities were observed.

\section{Laboratory abnormalities}

Tasquinimod led to a transient increase in laboratory parameters, such as white blood cell count, amylase, lipase, pancreatic amylase, CRP and fibrinogen, after 1-2 months of treatment; these parameters generally returned to normal within 6 months. The increased levels of amylase and lipase were asymptomatic. Increased levels of CRP correlated with an increased risk of AEs, such as muscle and joint pain. An increase in circulating VEGF levels was observed with tasquinimod treatment, perhaps indicating interference with VEGFR signaling, similar to that seen with sunitinib or a potential mechanism of therapeutic resistance.

\section{Dose reduction or termination}

The striking AEs observed in the tasquinimod group were gastrointestinal events, muscle and joint pain, and fatigue. The majority of AEs (89\% tasquinimod; 94\% placebo) were grade 1-2. Incidence of grade 4 or higher AEs were infrequent and did not differ between treatment groups. The most common grade 3-4 AEs in the tasquinimod group were increased lipase, muscular weakness, deep vein thrombosis, anemia, asthenia, renal failure and pneumonia. A minor decrease in mean blood pressure was seen in the tasquinimod group, and no corrected QT interval changes were noted. No difference in the number of arterial thrombotic events was observed between the arms (3 vs 3\%).

Dose reduction or termination for any reason during the first 9 weeks was documented in 55\% (74 out of 134) of tasquinimod-treated patients. Fifteen of the 31 patients who discontinued due to AEs, withdrew after experiencing grade $1-2$ toxicities. Discontinuation rate owing to toxicity was 22 versus $1 \%$ for patients receiving placebo, often occurring without protocol-specified dose reductions; the most common reason for treatment discontinuation owing to toxicity was muscle and/or joint pain.

For the majority of patients, tasquinimod had an acceptable toxicity profile, but in some patients, particularly men older than 75 years of age, tasquinimod was sufficiently poorly tolerated that patients or providers felt it necessary to stop therapy. Patients older than 80 years of age were more likely to develop AEs, with $73 \%$ (22 out of 30) requiring dose reduction or withdrawal during the first 9 weeks versus $50 \%$ of men younger then 80 years of age (52 out of 104).

\section{Tasquinimod in a Phase III trial for prostate cancer}

Based on the favorable Phase II trial results that showed that through the use of intrapatient stepwise dose escalation to individual MTDs, tasquinimod delayed disease progression in men with metastatic minimally symptomatic CRPC by a median of 4.3 months (without significant toxicities in the majority of men) a Phase III trial was initiated [102]. More than 1200 men were randomized in this Phase III clinical trial between tasquinimod or placebo for which accrual was completed at the end of 
2012. This global, pivotal trial was carried out in patients with mCRPC using a randomized, double-blind, placebo-controlled design. It will further evaluate the overall clinical benefit of tasquinimod in a larger predocetaxel mCRPC population. The aim of the study is to confirm tasquinimod's efficacy, with radiological PFS as the primary end point and OS as the secondary end point. The study recruited patients in more than 250 centers all over the world. Eligible patients with mCRPC were randomly assigned in a 2:1 ratio to one of two treatment groups: treatment group A (tasquinimod 0.25, 0.5 or $1 \mathrm{mg} / \mathrm{day} ; \mathrm{n}=800$ ) or treatment group $\mathrm{B}$ (placebo; $n=400)$. Patients were stratified by KPS ( $\geq 90$ vs $<90 \%$ ), presence or absence of visceral disease (all soft tissue except lymph nodes and local recurrence) and geographic region of enrollment (North America; Europe, Middle East and Africa; Asia-Pacific; and Latin America) to ensure evenness in treatment allocation within the specified levels of these factors, to avoid confounding the treatment effect. Patients in both treatment groups received once-daily oral dosing with study drug throughout the trial.

\section{Other ongoing trials with tasquinimod in prostate cancer}

\section{Switch maintenance trial}

The purpose of this randomized, double-blind, placebo-controlled proof-of-concept study is to evaluate and confirm that tasquinimod used as a maintenance therapy is active and tolerable in patients with advanced mCRPC not progressing after a first chemotherapy with docetaxel [103]. Prolongation of the response to first-line chemotherapy (consolidation) represents an unmet need for which patients and physicians request further therapeutic options. 'Switch maintenance' therapy with a manageable safety profile, such as tasquinimod treatment, could be one strategy to prolong treatment response, without significantly compromising quality of life.

The switch maintenance Phase II trial is a global, 1:1 randomized, placebo-controlled, double-blind Phase II trial investigating up to $1 \mathrm{mg} /$ day of tasquinimod versus placebo in 140 patients. The primary end point of the study is radiological PFS. The study will be recruiting across approximately 50 centers in Europe.

CATCH trial: tasquinimod plus cabazitaxel in chemotherapy-pretreated men with MCRPC

An early-stage, combination therapy trial (CATCH trial) of tasquinimod and cabazitaxel
(Jevtana $^{\circledR}$; Safoni, Paris, France) has been initiated for the treatment of men with heavily pretreated, mCRPC [104]. The Phase I trial will enroll up to 32 patients with mCRPC. Eligible patients must be refractory or intolerant to standard forms of chemotherapy. The trial is being conducted at two centers, the Duke Cancer Institute in Durham (NC, USA) and the University of Chicago (IL, USA). The primary objective of the study is to determine the appropriate dose of tasquinimod to be used in combination with cabazitaxel and prednisone based on safety and tolerability in men with chemorefractory mCRPC. As a secondary outcome, PFS will be assessed as a measure of efficacy of the drug. Preliminary evidence of durable efficacy will be based on a modified PCWG2-defined radiologic PFS, including response evaluation criteria in solid tumors group 1.1 criteria. Duration of PSA responses will be measured. In addition, changes of circulating tumor cells over time will be assessed (favorable changes in circulating tumor cell number $\geq 5$ to $<5$ ) and the proportion of men who achieve a reduction in CTC count will be a secondary end point.

\section{Ongoing trials with tasquinimod in other malignancies}

Other indications are currently under investigation in patients with other types of malignancies, for example, hepatocellular, ovarian, renal and gastric cancers $[105,106]$.

\section{Conclusion}

A myriad of novel agents are currently entering the treatment field of CRPC patients, spanning the early metastatic phase of CRPC to the more advanced postchemotherapy stage in patients with higher tumor burdens. The challenges in the coming years will include: optimal spacing of novel treatment strategies in this area by identifying and optimizing the most appropriate sequence of administration of these new agents as well as the combination of agents. Development of molecular biomarkers to better identify the patients who are likely to benefit from a particular agent will also be essential. Exploration of the utility of imaging techniques as predictive markers of efficacy will be crucial to minimize the time on treatment for those patients who will not respond to a particular agent.

\section{Future perspective}

Tasquinimod is a novel immunomodulatory agent with antiangiogenic activity that enhances the therapeutic anticancer response when 
combined with other standard-of-care modalities (radiation, androgen ablation and/or taxanebased chemotherapies) in experimental animal models. It has completed clinical Phase II testing in humans and has been tested in a Phase III setting.

It appears that patients with metastatic CRPC that are minimally symptomatic and have minimal tumor burden seem the most suitable group to use agents that target tumor angiogenesis rather than using cytotoxic chemotherapy. This tumor stage is more attractive for a blood vessel modulatory agent as compared with the more advanced stage with florid bone metastases. Thus, the Phase III trial with single agent tasquinimod could be positive in terms of adding to the OS. The disadvantage of this approach is that the results may take years before differences in OS are observed and we may have to rely on PFS and crossover. Until there is evidence to the contrary, it seems that these trials should utilize a

\section{Executive summary}

\section{Background}

- Angiogenesis has been accepted as a hallmark of cancer and inhibition of angiogenic pathways have shown to be an effective treatment strategy for the treatment of a number of solid tumors.

- Although angiogenesis is accepted to be key in prostate cancer, the efforts at blocking angiogenesis in the management of metastatic prostate cancer has so far been unsuccessful.

\section{Mechanism of action}

- Tasquinimod is a novel immunomodulatory agent with antiangiogenic activity and has, in particular, been tested against prostate cancer experimental animal models.

- The precise mechanism of action of tasquinimod is not precisely understood.

- Anti-tumor and antiangiogenic effects of tasquinimod are not fully elucidated but presumed to act via inhibition of TSP-1, an endogenous angiogenesis inhibitor, and inhibition of S100A9.

\section{Pharmacokinetic properties}

- In Phase I studies, tasquinimod could be safely administered at 0.5-1.0 mg per day during long-term treatment.

- The oral clearance was $0.28 \mathrm{l} / \mathrm{h}$ (mean) and the oral volume of distribution was $15 \mathrm{I}$ (mean), resulting in an elimination half-life of $40 \pm 16 \mathrm{~h}$ (mean \pm standard deviation). The area under the curve at steady state was $4.8 \mu \mathrm{mol} / \mathrm{h} / \mathrm{l}$ (mean).

\section{Safety \& tolerability}

- Side effects included transient elevation hyperamylasemia in the absence of clinical symptoms. The toxicity profile of tasquinimod is very different from that of tyrosine kinase (VEGFR) inhibitors and anti-VEGF targeting agents. Skin rashes, hand-foot skin reactions, diarrhea and hypertension were not observed, whereas an asymptomatic increase in serum levels of amylase and lipase has not been observed with tyrosine kinase inhibitors.

- The most frequently reported possibly or probably related adverse events ( $\geq 5 \%$ ) based on the Phase I and II studies were, in decreasing order: nausea, fatigue, headache, constipation, arthralgia, flatulence, diarrhea, decreased appetite, vomiting, myalgia, pain in extremity, anemia, back pain, increase in blood amylase and lipase.

- A low percentage of vascular events occurred, despite recent cardiovascular disease being an exclusion criterion in this elderly patient population.

- Patients older than 75 years of age may be more prone to develop nontolerable adverse events when treated with tasquinimod. A high percentage of patients stopped within 9 weeks of treatment, which may also be related to an older population with only minimal disease.

\section{Clinical efficacy}

- Tasquinimod significantly slowed progression and improved progression-free survival (PFS) in patients with metastatic castration-resistant prostate cancer (CRPC); it delayed PFS in men with metastatic minimally symptomatic chemotherapy-naive CRPC by a median of 4.3 months.

- PFS was improved across all prostate cancer working group CRPC subtypes of patients defined by localization of metastatic lesions (visceral metastases, bone metastases with or without nodal metastases, and lymph node only metastases). Among men with bone metastases only at baseline, median PFS was 12.1 versus 5.4 months (hazard ratio: $0.45 ; 95 \% \mathrm{Cl}: 0.23-0.88 ; p=0.016$ ).

- The general lack of prostate-specific antigen decline is compatible to what had been observed in CRPC using other antiangiogenic agents. Both preclinical and clinical data do not support the use of serum prostate-specific antigen as an indicator of therapeutic response.

\section{Phase III study}

- It will be important to further explore cardiac side effects in this large Phase III study.

- Of note, other classical antiangiogenic agents, such as sunitinib, bevacizumab, lenalidomide and aflibercept, have thus far shown some activity in Phase II trials, but no superiority when combined with docetaxel and measured against docetaxel in patients with more advanced CRPC. 
stepwise-dose-escalation-to-individual-MTD design, should exclude patients with a history of cardiovascular disease or pancreatitis, and study groups should be well balanced for age, pattern of metastasis, tumor pain and concomitant therapies. Future trials should also investigate prospective markers to predict susceptibility to cardiac and gastrointestinal (including pancreatic) side effects and the relationship between plasma concentrations and these effects.

\section{Financial \& competing interests disclosure}

The authors have no relevant affiliations or financial involvement with any organization or entity with a financial interest in or financial conflict with the subject matter or materials discussed in the manuscript. This includes employment, consultancies, honoraria, stock ownership or options, expert testimony, grants or patents received or pending, or royalties.

No writing assistance was utilized in the production of this manuscript.

\section{References}

Papers of special note have been highlighted as: - of interest

1. Ferlay J, Shin HR, Bray F et al. GLOBOCAN 2008, Cancer Incidence and Mortality Worldwide: No. 10. International Agency for Research on Cancer CancerBase, Lyon, France (2010).

2. Jemal A, Center MM, DeSantis C et al. Global patterns of cancer incidence and mortality rates and trends. Cancer Epidemiol. Biomarkers Prev. 19(8), 1893-1907 (2010).

3. Jemal A, Bray F, Center MM et al. Global cancer statistics. CA Cancer J. Clin. 61(2), 69-90 (2011).

4. American Cancer Society. Cancer Facts and Figures 2010. American Cancer Society, GA, USA (2010).

5. Osanto S, Van Poppel H. Emerging novel therapies for advanced prostate cancer. Ther Adv. Urol. 4(1), 3-12 (2012).

6. de Bono JS, Oudard S, Ozguroglu M et al. Prednisone plus cabazitaxel or mitoxantrone for metastatic castration-resistant prostate cancer progressing after docetaxel treatment: a randomised open-label trial. Lancet 376, $1147-1154$ (2010).

7. de Bono JS, Logothetis CJ, Molina A et al.; COU-AA-301 Investigators. Abiraterone and increased survival in metastatic prostate cancer. N. Engl. J. Med. 364, 1995-2005 (2011).

8. Kantoff PW, Higano CS, Shore ND et al. Sipuleucel-T immunotherapy for castrationresistant prostate cancer. N. Engl. J. Med. 363 , 411-422 (2010).

9. Ryan CJ, Smith MR, de Bono JS et al. Interim analysis results of COU-AA-302, a randomized, Phase III study of abiraterone acetate in chemotherapy-naive patients with metastatic castration-resistant prostate cancer. J. Clin. Oncol. 30(Suppl.), Abstract LBA4518 (2012).

10. Parker C, Nilsson S, Heinrich D et al.; ALSYMPCA Investigators. Alpha emitter radium-223 and survival in metastatic prostate cancer. N. Engl. J. Med. 369(3), 213-223 (2013).

11. Scher HI, Fizazi K, Saad F et al.; AFFIRM Investigators. Increased survival with enzalutamide in prostate cancer after chemotherapy. N. Engl. J. Med. 367(13), 1187-1197 (2012).

12. Ryan CJ, Smith MR, de Bono JS et al. Abiraterone in metastatic prostate cancer without previous chemotherapy. $N$. Engl. J. Med. 368, 138-148 (2013).

13. Greenblatt M, Shubi P. Tumor angiogenesis: transfilter diffusion studies in the hamster by the transparent chamber technique. J. Natl Cancer Inst. 41(1), 111-124 (1968).

14. Ehrmann RL, Knoth M. Choriocarcinoma. Transfilter stimulation of vasoproliferation in the hamster cheek pouch. Studied by light and electron microscopy. J. Natl Cancer Inst. 41(6), 1329-1341 (1968).

15. Folkman J. Tumor angiogenesis: therapeutic implications. N. Engl. J. Med. 285(21), 1182-1186 (1971).

- Excellent preclinical pioneering study highlighting the importance of angiogenesis for tumor growth and autocrine proangiogenic factors.

16. Gullino PM. Angiogenesis and oncogenesis. J. Natl Cancer Inst. 61(3), 639-643 (1978).

17. Bouck N, Stellmach V, Hsu SC. How tumors become angiogenic. Adv. Cancer Res. 69, 135-174 (1996).

18. Hanahan D, Weinberg RA. The hallmarks of cancer. Cell 100(1), 57-70 (2000).

19. Vukanovic J, Passaniti A, Hirata T et al. Antiangiogenic effects of the quinoline-3carboxamide linomide. Cancer Res. 53(8), 1833-1837 (1993).

20. Ichikawa T, Lamb JC, Christensson PI et al. The anti-tumor effects of the quinoline-3carboxamide linomide on Dunning R-3327 rat prostatic cancers. Cancer Res. 52(11), 3022-3028 (1992).

21. Isaacs JT, Pili R, Qian DZ et al. Identification of ABR-215050 as lead second generation quinoline-3-carboxamide antiangiogenic agent for the treatment of prostate cancer. Prostate 66(16), 1768-1778 (2006).

22. Dalrymple SL, Becker RE, Isaacs JT. The quinoline-3-carboxamide antiangiogenic agent, tasquinimod, enhances the anti-prostate cancer efficacy of androgen ablation and taxotere without effecting serum PSA directly in human xenografts. Prostate 67(7), 790-797 (2007).

23. Olsson A, Björk A, Vallon-Christersson J et al. Tasquinimod (ABR-

215050), a quinoline-3-carboxamide antiangiogenic agent, modulates the expression of thrombospondin-1 in human prostate tumors. Mol. Cancer 9, 107-120 (2010).

24. Dalrymple SL, Becker RE, Zhou $\mathrm{H}$ et al. Tasquinimod prevents the angiogenic rebound induced by fractionated radiation resulting in an enhanced therapeutic response of prostate cancer xenografts. Prostate 72(6), 638-648 (2012).

25. Jennbacken $\mathrm{K}$, Welén $\mathrm{K}$, Olsson A et al. Inhibition of metastasis in a castration resistant prostate cancer model by the quinoline-3-carboxamide tasquinimod (ABR-215050). Prostate 72(8), 913-924 (2012).

26. Isenberg JS, Martin-Manso G, Maxhimer JB et al. Regulation of nitric oxide signalling by thrombospondin 1: implications for anti-angiogenic therapies. Nat. Rev. Cancer 9(3), 182-194 (2009).

27. Rafii S, Lyden D. S100 chemokines mediate bookmarking of premetastatic niches. Nat. Cell Biol. 8(12), 1321-1323 (2006).

28. Sinha P, Okoro C, Foell D et al. Proinflammatory S100 proteins regulate the accumulation of myeloid-derived suppressor cells. J. Immunol. 181(7), 4666-4675 (2008).

29. Cheng P, Corzo CA, Luetteke $\mathrm{N}$ et al. Inhibition of dendritic cell differentiation and accumulation of myeloid-derived suppressor cells in cancer is regulated by S100A9 protein. J. Exp. Med. 205(10), 2235-2249 (2008). 
30. Turovskaya O, Foell D, Sinha P et al. RAGE, carboxylated glycans and S100A8/A9 play essential roles in colitis-associated carcinogenesis. Carcinogenesis 29(10), 2035-2043 (2008).

31. Källberg E, Vogl T, Liberg D et al. S100A9 interaction with TLR4 promotes tumor growth. PLoS One 7(3), e34207 (2012).

32. Isaacs JT, Antony L, Dalrymple SL et al. Tasquinimod is an allosteric modulator of HDAC4 survival signaling within the compromised cancer microenvironment. Cancer Res. 73(4), 1386-1399 (2013).

33. Björk P, Björk A, Vogl T et al. Identification of human S100A9 as a novel target for treatment of autoimmune disease via binding to quinoline-3-carboxamides. PLoS Biol. 7(4), e97 (2009).

34. Bratt $\mathrm{O}$, Häggman $\mathrm{M}$, Ahlgren $\mathrm{G}$ et al. Open-label, clinical Phase I studies of tasquinimod in patients with castrationresistant prostate cancer. Br. J. Cancer 101(8), 1233-1240 (2009).

35. Pili R, Häggman M, Stadler WM et al. Phase II randomized, double-blind, placebo-controlled study of tasquinimod in men with minimally symptomatic metastatic castrate-resistant prostate cancer. J. Clin. Oncol. 29(30), 4022-4028 (2011).
- Relevant data from a pivotal Phase II clinical trial with regards to the occurrence of side effects, minimal prostate-specific antigen response and clinical efficacy.

36. Scher HI, Halabi S, Tannock I et al. Design and end points of clinical trials for patients with progressive prostate cancer and castrate levels of testosterone: recommendations of the Prostate Cancer Clinical Trials Working Group. J. Clin. Oncol. 26(7), 1148-1159 (2008).

37. Hansson GP, Olin M, Svanström C et al. Bioanalysis in clinical development of tasquinimod using liquid chromatography/ tandem mass spectrometry.

J. Chromatogr. B Analyt. Technol. Biomed. Life Sci. 879(30), 3401-3406 (2011).

\section{Websites}

101. Ferlay J, Shin HR, Bray F, Forman D, Mathers C, Parkin DM. GLOBOCAN 2008, cancer incidence and mortality worldwide: IARC CancerBase No 10 (2010).

http://globocan.iarc.fr

102. ClinicalTrials.gov. A Study of Tasquinimod in Men with Metastatic Castrate Resistant Prostate Cancer. Primary Outcome Measures: a Phase 3 Randomized, Double-Blind,
Placebo-Controlled Study of Tasquinimod in Men with Metastatic Castrate Resistant Prostate Cancer.

http://clinicaltrials.gov/ct2/show/ NCT01234311?term=tasquinimod $\&$ rank=3

103. ClinicalTrials.gov. The CATCH Prostate Cancer Trial: Cabazitaxel and Tasquinimod in Men with Prostate Cancer. http://clinicaltrials.gov/ct2/show/ NCT01513733?term=tasquinimod\&rank=1

104. ClinicalTrials.gov. A Proof of Concept Study of Maintenance Therapy with Tasquinimod in Patients with Metastatic Castrate-resistant Prostate Cancer Who are not Progressing After a First Line Docetaxel Based Chemotherapy. http://clinicaltrials.gov/ct2/show/ NCT01732549? term=tasquinimod $\&$ rank= 4

105. ClinicalTrials.gov. A Study of Tasquinimod in Men with Metastatic Castrate Resistant Prostate Cancer. http://clinicaltrials.gov/ct2/show/ NCT01234311

106. ClinicalTrials.gov. A Study with Tasquinimod Treating Patients in Four Independent Cohorts of Hepatocellular, Ovarian, Renal Cell and Gastric Cancers. www.clinicaltrials.gov/ct2/show/ NCT01743469 